\title{
CdTe x-ray sensing driven by electron beam from field emitters
}

\author{
Yoichiro Neo, ${ }^{\text {a) }}$ Yoshiaki Ikeda, Takuya Sakata, Hisashi Morii, Kazufumi Shiozawa, \\ Toru Aoki, and Hidenori Mimura \\ Research Institute of Electronics, Shizuoka University, 3-5-1 Johoku, Hamamatsu 432-8011, Japan
}

(Received 17 July 2006; accepted 11 December 2006; published 30 March 2007)

\begin{abstract}
The authors have demonstrated a novel CdTe x-ray sensing device that consists of a CdTe $p n$ diode and a graphite nanoneedle field emitter. The CdTe diode was fabricated using an excimer laser doping method. The signal current was successfully read out by an electron beam from the field emitter and was found to be proportional to the x-ray intensity. The operation principle of the device is expounded in this article. (c) 2007 American Vacuum Society. [DOI: 10.1116/1.2432356]
\end{abstract}

\section{INTRODUCTION}

A photon-counting mode CdTe x-ray imaging device with an energy discrimination function has attracted much interest because of its high sensitivity and energy discrimination. ${ }^{1,2} \mathrm{~A}$ CdTe imaging device has been developed, consisting of 4 $\times 128$ structured 512 superlinear CdTe detectors with a pixel size of $0.8 \times 2 \mathrm{~mm}^{2}$ and a pixel separation of $0.2 \mathrm{~mm}$. With imaging devices, it is important to reduce the pixel size and arrange the pixels in a high-density array for high spatial resolution images. However, smaller pixel size and higherdensity arrangements are difficult to produce with CdTe devices, because the fineness of the process significantly degrades the device performance. Recently, the imaging sensor consisting of a high-gain avalanche rushing amorphous photoconductor (HARP) target and a field emitter array (FEA), named as a FEA HARP image sensor, has been reported and high-resolution images were demonstrated. ${ }^{4,5}$ The basic operating principle is similar to that of a camera tube, and the image signal is obtained by sequentially recombining the electrons emitted from the FEA with the charge (hole) pattern, which is optically generated and accumulated on the HARP target. It is not necessary in this device that each pixel is separated for high resolution. Therefore, if a CdTe x-ray sensor (target) were to be scanned by an electron beam from a FEA, similar to the FEA HARP image sensor, highresolution images should be expected. As the first step, the operation principle of the device is verified. Besides, two kinds of electron landing material were compared and the influence of secondary electron yield was demonstrated in this article.

\section{EXPERIMENTS}

Cl-doped $p$-type ( $\pi$-type) single-crystal CdTe (111) wafers with a high resistivity, of the order of $10^{9} \Omega \mathrm{cm}$, were used in the experiments. $M-\pi-n$ structured CdTe diodes were fabricated by excimer laser doping. A thin indium layer was evaporated on the $p$-type CdTe as a $n$-type dopant material. Then, a $n$-type CdTe layer was formed by irradiating the dopant layer with a $\mathrm{KrF}$ excimer laser in a high-pressure

\footnotetext{
a) Author to whom correspondence should be addressed; electronic mail:
} y-neo@rie.shizuoka.ac.jp argon ambient. The details of fabrication processes have been reported previously. ${ }^{6} \mathrm{~A}$ thin $\mathrm{Sb}_{2} \mathrm{~S}_{3}$ film was evaporated on the $p$-type side of the CdTe diode as a landing material for the electron beam.

The cathode used in this experiment was composed of sputter-induced graphite nanoneedle field emitters. These nanoneedle field emitters were fabricated using conventional rf magnetron sputtering equipment. A graphite rod, $1 \mathrm{~mm}$ in diameter, was positioned on the cathode electrode and was sputtered in $\mathrm{a}_{2}$ gas ambient at a pressure of $30 \mathrm{~Pa}$, at a power of $600 \mathrm{~W}$, for a sputtering time of $30 \mathrm{~min}$. The emitter was composed of an extremely large number of nanoneedles, whose apexes and height were less than $50 \mathrm{~nm}$ and $3-5 \mu \mathrm{m}$, respectively. ${ }^{7}$ The graphite nanoneedle emitter exhibited a high brightness of about $10^{11} \mathrm{Asr}^{-1} \mathrm{~m}^{-2}$ and could operate at residual pressure as high as $10^{-5}-10^{-4} \mathrm{~Pa}^{7}$

Figure 1 shows a schematic of a CdTe x-ray sensing device using an electron beam for the verification of the operating principle. The cathode and CdTe diode were set in the center of the chamber. The cathode consisted of a graphite nanoneedle field emitter and an extraction gate electrode fabricated from a $\mathrm{Cu}$ mesh. The separation of the emitter and the gate was $0.1 \mathrm{~mm}$. The CdTe diode was located a few millimeters above the cathode and its $p$-type side faced the cathode. All experiments were carried out at an ultrahigh vacuum of about $5 \times 10^{-6} \mathrm{~Pa}$. The graphite nanoneedle field emitter was maintained at $0 \mathrm{~V}$ and the extraction gate electrode was biased up to $1100 \mathrm{~V}$ to obtain field emission. The emission current was about $3 \mu \mathrm{A}$ at a gate voltage of $1100 \mathrm{~V}$. A positive voltage from 0 to $200 \mathrm{~V}$ was applied to the $n$-type region of the CdTe diode. $\mathrm{X}$ rays were introduced through a viewing port on the chamber.

\section{RESULTS AND DISCUSSIONS}

Figure 2 shows the output currents resulting from irradiation by an electron beam, with and without x-ray exposure. It is clear that the CdTe diode is maintained in a reverse biased state and that a signal current is obtained under the x-ray irradiation. This result implies that the total yield $\delta_{\text {eff }}$ [the summation of secondary electron (SE) yield and backscattering electron (BSE) yield] is less than unity and that the $p$-type region possesses a negative potential with respect to the $n$-type CdTe side. Therefore, the CdTe diode is reverse 


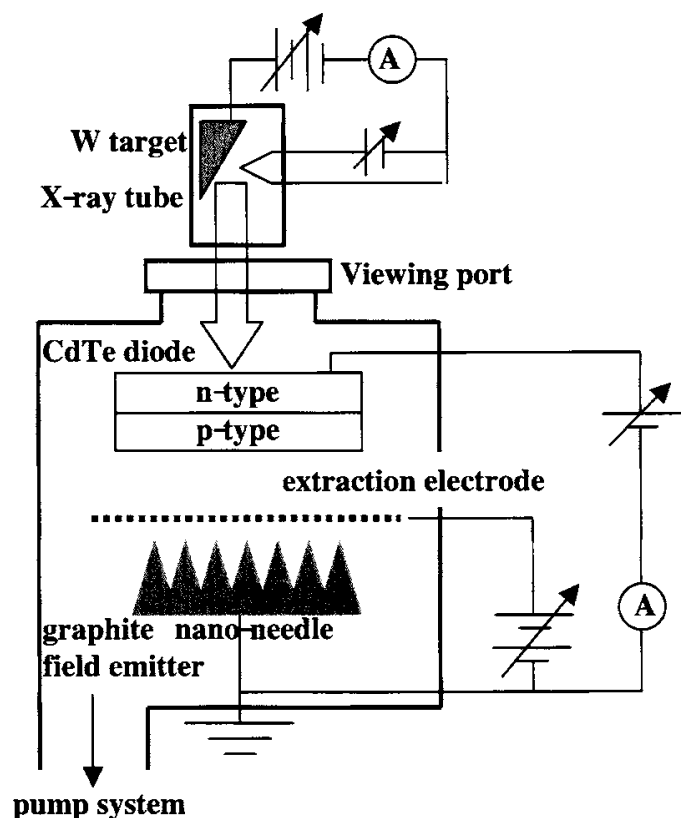

FIG. 1. Schematic of a CdTe x-ray sensing device using a graphite nanoneedle cathode.

biased and a depletion layer spreads into the $p$-type region. In this situation, the charges (holes), which are generated by $\mathrm{x}$-ray, drift and are accumulated on the surface of the CdTe diode. The accumulated holes recombine with electrons supplied from the graphite nanoneedle field emitter. As a result, the signal current, which depends on the x-ray intensity, is effectively detected by electron beam.

Figure 3 shows the dependence of the signal current on the emission current from the x-ray tube at a CdTe bias voltage of $20 \mathrm{~V}$. The $\mathrm{x}$ ray was pulsed and the hatched areas correspond to the $\mathrm{x}$-ray exposure. The intensity of $\mathrm{x}$ rays was decreased by decreasing the emission current of the $\mathrm{x}$-ray tube from $110 \mu \mathrm{A}$ to zero in $10 \mu \mathrm{A}$ steps. As shown in Fig. 3 , the signal current decreases with the decrease in the $\mathrm{x}$-ray tube current. The inset shows the relationship between the

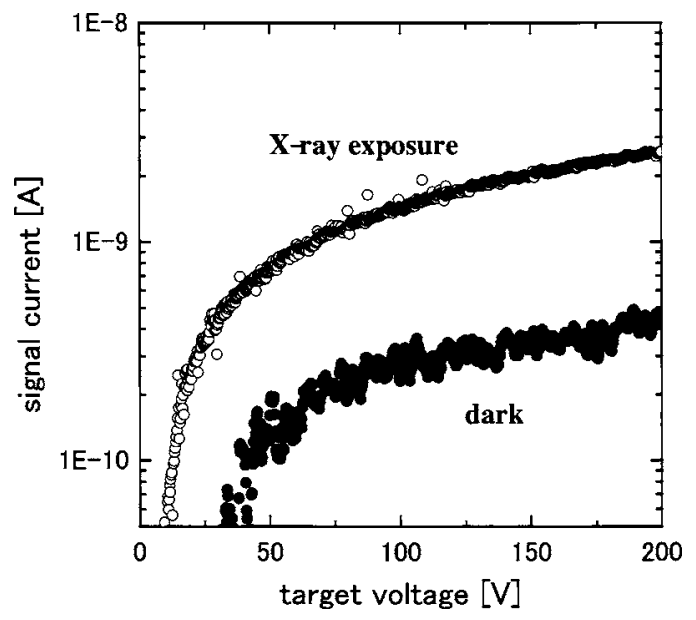

FIG. 2. Output currents under electron beam irradiation, with and without $\mathrm{x}$-ray exposure.

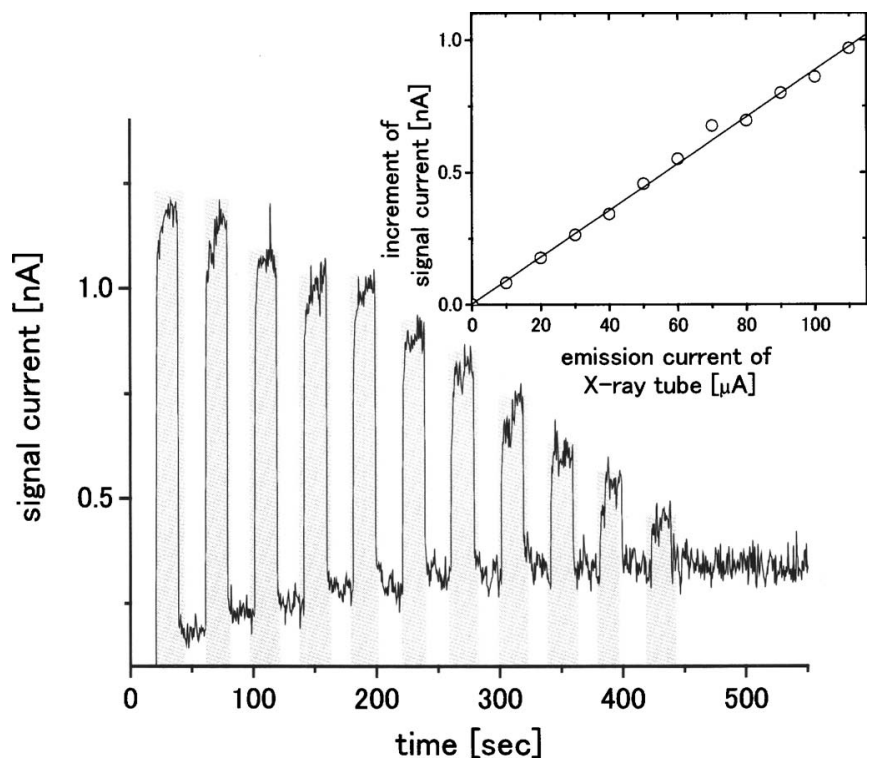

FIG. 3. Dependence of the output current on the x-ray intensity at a CdTe bias voltage of $20 \mathrm{~V}$. The hatched areas correspond to the x-ray exposure. The inset shows the relationship between the signal currents and the x-ray tube current.

signal currents and the $\mathrm{x}$-ray tube current. The signal current is clearly proportional to the $\mathrm{x}$-ray tube current, which is also proportional to the $\mathrm{x}$-ray intensity.

In a practical CdTe sensing device, the signal current would be read out by scanning the electron beam. Therefore, we measured the output current characteristic under x-ray exposure, with and without electron beam irradiation. Figure 4 shows the measurement results at a CdTe bias voltage of $20 \mathrm{~V}$. The hatched areas correspond to the electron beam irradiation. As shown in Fig. 4, the signal current is obtained only under electron beam irradiation.

To investigate the influence of the secondary electron yield, we replaced $\mathrm{Sb}_{2} \mathrm{~S}_{3}$ with $\mathrm{Au}$ as the electron landing material. Figure 5 shows the output currents under electron beam irradiation, with and without x-ray exposure for the $\mathrm{CdTe}$ diode with a $\mathrm{Au}$ electron landing material. When the

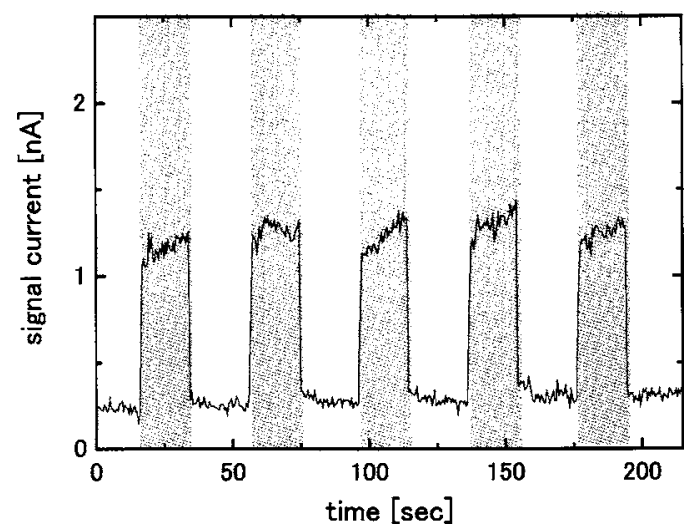

FIG. 4. Output current characteristic under x-ray exposure, with and without the irradiation by an electron beam, at a CdTe bias voltage of $20 \mathrm{~V}$. The hatched areas correspond to the electron beam irradiation. 


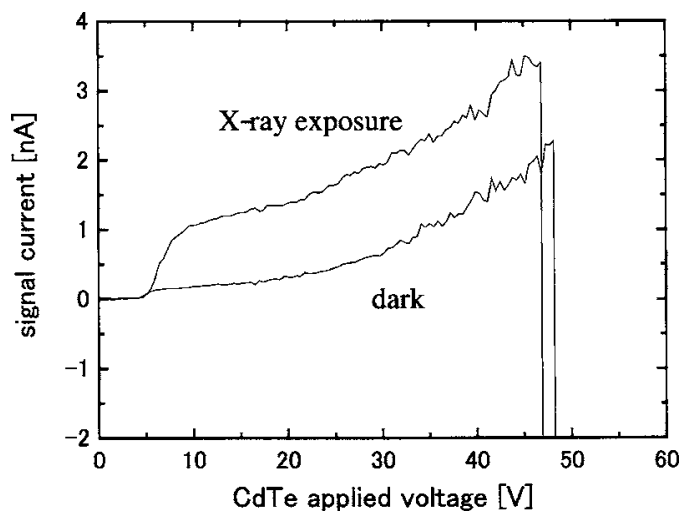

FIG. 5. Output currents under electron beam irradiation, with and without $\mathrm{x}$-ray exposure, for the $\mathrm{CdTe}$ diode with a Au electron landing material.

bias voltage applied to the CdTe diode is increased to about $50 \mathrm{~V}$, the polarity of signal current suddenly reversed. This polarity inversion implies that the total yield (SE+BSE) exceeds unity at a bias voltage above $50 \mathrm{~V}$.

Figure 6 shows the energy band diagrams of the CdTe diode with a Au electron landing material, deduced from the result in Fig. 5. When the bias voltage is less than about $50 \mathrm{~V}$, the total yield is less than 1.0 , the CdTe diode is reverse biased, and holes are accumulated at the surface of the

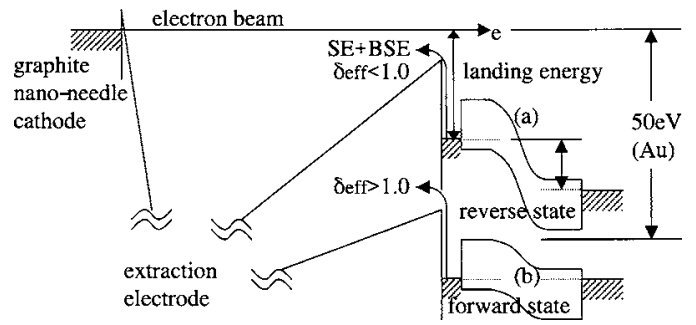

FIG. 6. Energy diagrams for the CdTe diode with a Au electron landing material. $\delta_{\text {eff }}$ is the total yield. (a) The diode is reverse biased at a bias voltage less than $50 \mathrm{~V}$. (b) The diode is forward biased at a bias voltage greater than $50 \mathrm{~V}$.
CdTe diode. On the other hand, when the bias voltage is raised to above about $50 \mathrm{~V}$, the total yield becomes greater than 1.0 and the CdTe diode is forward biased. This result indicates that the total yield from $\mathrm{Au}$ is much higher than that from $\mathrm{Sb}_{2} \mathrm{~S}_{3}$.

\section{CONCLUSION}

We have demonstrated a novel CdTe x-ray sensing device that consists of a CdTe $p n$ diode and a field emitter. The $\mathrm{CdTe}$ diode was fabricated using an excimer laser doping method and the field emitter used in this experiment was composed of sputter-induced graphite nanoneedle field emitters. During the device operation, the CdTe diode is reverse biased and the holes, which are generated by $\mathrm{x}$ ray, drifted and are accumulated on the surface of the CdTe diode. The signal current is successfully detected by the recombination of holes with electrons from the field emitter. The electron landing material significantly influences the current-voltage characteristics, which can be ascribed to the total yield of the material.

\section{ACKNOWLEDGMENTS}

The authors would like to thank S. Yamashita and K. Matsubara for their technical assistance. This work was supported, in part, by Shizuoka University 21st COE (Center of Excellence) Program from the Ministry of Education, Culture, Sports, Technology and Science, Japan.

${ }^{1}$ T. Aoki, Y. Ishida, Y. Makino, G. Ohashi, Y. Tomita, H. Morii, J. Temmyo, and Y. Hatanaka, Proceedings of IDW/AD'05, 2005 (unpublished), p. 4283.

${ }^{2}$ T. Aoki, A. Nakamura, M. Niraula, Y. Tomita, and Y. Hatanaka, Proc. SPIE 4784, 259 (2002).

${ }^{3}$ D. Furuhashi, D. Sakashita, Y. Ishida, T. Aoki, Y. Tomita, Y. Hatanaka, and J. Temmyo, Proc. SPIE 5922, 184 (2005).

${ }^{4}$ N. Egami et al., J. Vac. Sci. Technol. B 23, 2056 (2005).

${ }^{5}$ N. Negishi et al., J. Vac. Sci. Technol. B 24, 1021 (2006).

${ }^{6}$ T. Aoki, V. Gnatyuk, A. Nakamura, Y. Tomita, Y. Hatanaka, and J. Temmyo, Phys. Status Solidi C 1, 1050 (2004).

${ }^{7}$ Y. Neo, H. Mimura, and T. Matsumoto, Appl. Phys. Lett. 88, 073511 (2006). 